lateral forces can then be controlled by the optical spin.

Polarization gratings can be seen as space-variant (transversely inhomogeneous) Pancharatnam-Berry-phase optical elements (PBOEs) $)^{12}$, where the state of polarization of the incoming beam is modulated by means of a subwavelength-period carrier grating with a periodically changing fringe orientation. Interestingly, by continuously controlling the local orientation and period of the grating, it is possible to achieve any desired phase element ${ }^{12}$. The same idea can be extended to designed 'metasurfaces' or other optical elements not necessarily based on PBOEs. The appropriate combination of different metasurfaces on a given object could open the possibility of light-spin control of the motion of an optically driven macroscopic body far from the light sources. For example, the same polarization grating illuminated by two beams at different angles (Fig. 2c) will deflect the incoming beams along the vertical axis. Momentum conservation would now result in a 'negative' pulling force on the macroscopic object ${ }^{3}$.

Metasurfaces can also be designed to generate orbital angular momentum and are being routinely used as compact vortex generators. It is possible to design them to induce a large orbital angular momentum to an incoming circularly polarized planewave. In analogy with the discussion of linear momentum transfer, the conservation of total angular momentum results in a torque on the sample whose magnitude and sign can be manipulated by the specific design of the metasurface. With this in mind, Magallanes and Brasselet addressed the problem of the measurement of lefthanded torques by direct observation of the light-induced spinning of the sample. Left-handed torques have been indirectly measured by analysing the rotational Doppler frequency shift of the scattered light ${ }^{6}$. The researchers' simple design of the sample allows the sample rotation to be observed in a sense of rotation opposite to that prescribed by the spin angular momentum of the incident light ${ }^{9}$.

Apart from suggesting new research paths, the researchers' results could open the possibility of building macroscopic light-driven meta-machines, with specifically designed metasurfaces, possibly reconfigurable, for complex mechanical tasks involving either translational or rotational degrees of freedom, or a combination thereof. The elegant conceptual simplicity of the researchers' work and the direct and unambiguous observation of spin-dependent optical forces and lefthanded torques on macroscopic objects with the naked eye can be considered ground-breaking in the perception of optomechanical problems.

Jorge Olmos-Trigo and Juan José Sáenz* Donostia International Physics Center (DIPC),

Donostia-San Sebastián, Spain.

*e-mail: juanjo.saenz@dipc.org

Published online: 27 July 2018

https://doi.org/10.1038/s41566-018-0223-3

References

1. Allen, L., Barnett, S. M. \& Padgett, M. J. Optical Angular Momentum (IOP Publishing, Bristol, 2003).

2. Glückstad, J. Nat. Photon. 5, 7-9 (2011).

3. Dogariu, A., Sukhov, S. \& Sáenz, J. J. Nat. Photon. 7, 24-27 (2013).

4. Chen, J., Ng, J., Lin, Z. \& Chan, C. T. Nat. Photon. 5, 531-534 (2011).

Brzobohaty, O. et al. Nat. Photon. 7, 123-127 (2013).

6. Hakobyan, D. \& Brasselet, E. Nat. Photon. 8, 610-614 (2014).

7. Sukhov, S. et al. Nat. Photon. 9, 809-812 (2015).

8. Antognozzi, M. et al. Nat. Phys. 10, 731-735 (2016).

9. Magallanes, H. \& Brasselet, E. Nat. Photon. https://doi.

org/10.1038/s41566-018-0200-x (2018).

10. Gori, F. Opt. Lett. 24, 584-586 (1999).

11. Tervo, J. \& Turunen, J. Opt. Lett. 25, 785-786 (2000).

12. Bomzon, Z., Biener, G., Kleiner, V. \& Hasman, E. Opt. Lett. 27, $1141-1143(2002)$

\title{
GRAPHENE
}

\section{Adaptive infrared camouflage}

Active thermal surfaces that can be electronically switched between states of high and low infrared emissivity have been made by a team of researchers from Turkey, the US and the UK (Salihoglu, O. et al. Nano Lett. 18, 4541-4548; 2018). The team says that the electronically controllable graphene surfaces can camouflage the thermal signature of an object they cover so that it blends in with background temperatures and becomes invisible to an infrared thermal camera. The surfaces can also make hot objects appear cold and vice versa.

The surfaces are made from multilayer graphene on top of an ionic-liquid-soaked polyethylene membrane and a thin bottom layer of gold. On application of an electrical bias of $\sim 3 \mathrm{~V}$ between the graphene and the gold, intercalation of positive ions into the graphene occurs. This serves to dope the graphene and increase the charge density and raise the Fermi level to higher energies, thus suppressing infrared absorption and emissivity. The result is that the surface

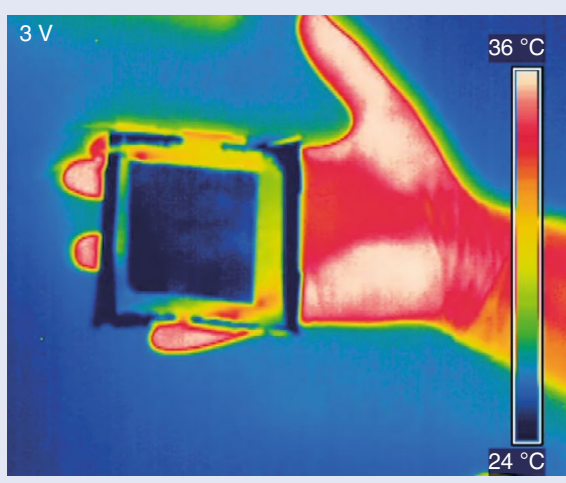

Credit: American Chemical Society

switches to an effective cold state. The effect is reversible and switching off the electrical bias causes the emissivity of the surface to rise back up to its original value.

Tests with the surface placed on a hot plate at a temperature of $55^{\circ} \mathrm{C}$ show the emissivity at a wavelength of $10 \mu \mathrm{m}$ changes between values of 0.76 to 0.33 as the voltage is ramped up from $0 \mathrm{~V}$ to
3.5 $\mathrm{V}$. The switching takes $<1 \mathrm{~s}$ and is accompanied by a step-like variation in the electrical sheet resistance from $33 \Omega$ to $0.6 \Omega$.

Furthermore, by pixelating the rear gold electrode, the surface can be turned into an array of independently controllable thermal pixels, allowing spatial temperature patterns to be created on demand. In experiments with a $5 \times 5$ array of square pixels (each with an area of $2 \times 2 \mathrm{~cm}^{2}$ ), the researchers were able to write the word 'HELLO' with a temperature contrast of $\sim 10^{\circ} \mathrm{C}\left(35-45^{\circ} \mathrm{C}\right)$.

As the surfaces are lightweight $\left(30 \mathrm{~g} \mathrm{~m}^{-2}\right)$, thin $(50 \mu \mathrm{m}$ thick), flexible and can potentially be fabricated in large areas, the team believes that a wide range of potential applications are possible, including their use in thermal camouflage and adaptive heat shields for satellites.

Oliver Graydon

Published online: 27 July 2018

https://doi.org/10.1038/s41566-018-0233-1 